\title{
Next frontiers in systemic therapy for soft tissue sarcoma
}

\author{
Cheuh-Chuan Yen ${ }^{1}$, Tom Wei-Wu Chen ${ }^{2,3,4}$ \\ ${ }^{1}$ Division of Medical Oncology and Center for Immuno-oncology, Department of Oncology, Taipei Veterans General Hospital, Taipei, Taiwan; \\ ${ }^{2}$ Department of Oncology, National Taiwan University Hospital, Taipei, Taiwan; ${ }^{3}$ Graduate Institute of Oncology, National Taiwan University \\ College of Medicine, Taipei, Taiwan; ${ }^{4}$ National Taiwan University Cancer Center, Taipei, Taiwan \\ Contributions: (I) Conception and design: All authors; (II) Administrative support: All authors; (III) Provision of study materials or patients: All \\ authors; (IV) Collection and assembly of data: All authors; (V) Data analysis and interpretation: All authors; (VI) Manuscript writing: All authors; (VII) \\ Final approval of manuscript: All authors. \\ Correspondence to: Tom Wei-Wu Chen, MD. Department of Oncology, National Taiwan University Hospital, 7 Chung-Shan South Rd, Taipei, \\ Taiwan. Email: saxotomy@gmail.com.
}

\begin{abstract}
Soft tissue sarcoma (STS) is a heterogeneous disease with more than 50 subtypes. Once the disease reached locally advanced or metastatic status, the standard treatment remains to be chemotherapy. Current understanding of the underlying molecular and genomic mechanisms of different histology subtypes have led to encouraging development of new drugs in treating STS. Besides molecular targeted therapy, immunotherapy have also shown promising advancement in solid tumor treatments. This review will be in two parts. The first part will focus on the molecular targeted agents aiming at molecular or genetic alterations that are more specific in STS, including antiangiogenic molecules, plate-derived growth factor receptor alpha (PDGFRA) monoclonal antibody, colony-stimulating factor-1 receptor (CSF-1R), selective inhibitors of nuclear export (SINE), cyclin-dependent kinase 4/6 (CDK 4/6), mdm2, and epigenetic regulators. We also discussed in depth about how current precision medicine influences the treatment paradigm in STS. In the second part, we focus on the landscape of immunotherapy in STS including immune checkpoint inhibitors (ICIs) and the combinations of immunotherapies or with other molecules that could modulate the tumor microenvironment. These included the program cell death-1 receptor and its ligand (PD-1/PD-L1), cytotoxic T lymphocyte associated protein-4 (CTLA-4) and the combination with anti-angiogenic agents that could facilitate the trafficking of T cells. Strategies targeting the tumor-associated antigen NY-ESO-1, which is commonly observed in synovial sarcoma and myxoid round cell liposarcoma, via viral vaccines and adoptive $\mathrm{T}$ cells will also be discussed. These new frontiers of treatment that are developed with better insights into sarcoma and immune biology hopefully will change the treatment paradigm of advanced STS in the future.
\end{abstract}

Keywords: Soft tissue sarcoma (STS); molecular targeted therapy; precision medicine; immunotherapy

Submitted Jul 13, 2018. Accepted for publication Aug 07, 2018.

doi: $10.21037 /$ cco.2018.08.04

View this article at: http://dx.doi.org/10.21037/cco.2018.08.04

\section{Introduction}

Soft tissue sarcomas (STSs) accounts for less than $1 \%$ of all cancer, but are highly heterogeneous in terms of anatomical location, histology, molecular characteristics and prognosis (1). Approximately $50 \%$ to $60 \%$ of cases occur in the extremities. Neoadjuvant and/or adjuvant chemotherapy or radiotherapy can be given, depending on the tumor histological subtype, grade, and margin status (2,3). A 5-year distant metastasis-free survival of over $60 \%$ can be achieved $(4,5)$. On the other hand, retroperitoneal sarcoma (RS) accounts for $15 \%$ of all STSs. Complete surgical resection with a negative margin is hard to achieve mostly because the tumor is deeply seated in the retroperitoneum adjacent to many vital organs, and oftentimes presents as multifocal disease (6). Local recurrence is the major cause of treatment 
failure in RS patients, and although retrospective study suggested radiation may play a role in disease control (7), the result of the prospective clinical trial [Surgery With or Without Radiation Therapy in Untreated Nonmetastatic Retroperitoneal Sarcoma (STRASS); NCT01344018] to understand the role of radiotherapy in improving local control of RS patients is highly anticipated.

For metastatic disease, chemotherapy is still the mainstream therapy. Single agent anthracycline is the standard first-line therapy, with a median overall survival around 12 to 14 months. Combination with ifosfamide may improve response rate but is associated with excessive toxicities (8). Gemcitabine plus docetaxel may be an alternative option (9). For the second line treatment, eribulin and trabectedin have demonstrated efficacy in liposarcoma (LPS) and leiomyosarcoma (LMS) $(10,11)$.

Progress of the treatment of advanced STS mainly comes from the understanding of driver oncogenes. Most of the gastrointestinal stromal tumors (GISTs) contained either $c$-KIT or PDGFRA mutation, which can be effectively inhibited by tyrosine kinase inhibitors (TKIs) such as imatinib, sunitinib or regorafenib (12-14). On the other hand, more than $85 \%$ of inflammatory myofibroblastic tumors (IMT) harbored kinase fusions involving $A L K$, ROS1, or PDGFR $\beta$ (15). ALK inhibitor, such as crizotinib, has shown potent efficacy in this disease (16). Other examples include imatinib or sunitinib for dermatofibrosarcoma protuberans harboring $\mathrm{t}$ $(17 ; 22)(\mathrm{q} 22 ; \mathrm{q} 13.1)$ translocation with resultant fusion gene COL1A1-PDGFB (17), or mTOR inhibitor for perivascular epithelioid cell tumor (PEComa) family with deletion or under-expression of TSC1 or TSC2 (18). The new frontiers of systemic treatments in advanced STS will come from not only the search of driver oncogenes in different STS histology but also the insight of other genomic, epigenetic, and immunological niches in STS. In this review, we will discuss recently identified novel treatments in STS based on different molecular and immune system pathways (Table 1).

\section{The molecular targeted therapies}

\section{Anti-angiogenic therapies}

Angiogenesis is one of the important hallmarks in cancer (44). Vascular endothelial growth factor (VEGF) is considered one of the main driving molecular for angiogenesis, and thus the main target for drug development. VEGF is found to be highly expressed in many types of STS (45), and the increased VEGF or other angiogenic factors are associated with a poor prognosis (46-48). Moreover, in comparison with healthy individuals, STS patients were found to have significantly elevated VEGF serum levels $(49,50)$. These studies hence provided the rationale for developing anti-angiogenesis therapy for STS.

The small-molecule VEGF inhibitor pazopanib is a multi-targeted TKI, with significant activity against receptor of VEGF type 1, 2, and 3 (VEGFR-1, -2, and -3), and platelet-derived growth factors (PDGFR) (51). In a stratified phase 2 clinical trial in relapsed or metastatic STS, except for LPS, it achieved a three-months progressionfree rate at around $40 \%$ in nearly all types of STS (19). In a subsequent randomized phase III study of patients with STS who failed standard chemotherapy, pazopanib demonstrated a superior median progression-free survival (PFS) than placebo control (4.6 vs. 1.6 months). Several other VEGFR TKIs such as regorafenib and sorafenib have also showed efficacy in STSs in phase II studies $(20,52)$.

Alveolar soft part sarcoma (ASPS) is a rare tumor accounting for less than $1 \%$ of STS and occurs primarily in young adults. It has an indolent clinical course, but is highly metastatic and frequently affects lungs, brain, and bones. In patients with advanced disease, the median survival is around 40 months and a 5 -year survival rate of $20 \%(53,54)$. Standard cytotoxic chemotherapy regimens are typically ineffective (55). ASPS is associated with an unbalanced $t$ $(\mathrm{X}, 17)(\mathrm{p} 11 ; \mathrm{q} 25)$ translocation, generating a ASPL-TFE3 chimeric transcription factor with resultant MET-related signal activation (56). Gene expression profiling of ASPS revealed upregulation of genes associated with angiogenesis as well (57). Several VEGFR TKIs have demonstrated their activities in ASPS $(58,59)$. In a phase II study of 43 patients with metastatic, unresectable ASPS, cediranib, another VEGFR TKI, resulted in a $35 \%$ of overall response rate (ORR). The disease control rate (partial response plus stable disease at 24 weeks) was $84 \%$ (36 of 43 patients). Gene profiling study revealed downregulation of genes related to vasculogenesis after treatment (21). Anti-angiogenesis therapy has also been shown efficacy in other rare STSs, such as desmoid tumor $(22,23)$, angiosarcoma (60) or solitary fibrous tumor/ hemangiopericytoma $(24,61)$.

\section{PDGFR- $\alpha$ monoclonal antibody}

Olaratumab is a fully human IgG1 antibody that selectively binds the PDGF $\alpha$-receptor and blocks ligand-induced 
Table 1 The list of new therapeutic agents in soft tissue sarcoma with their respective therapeutic targets and preferentially-targeted histology

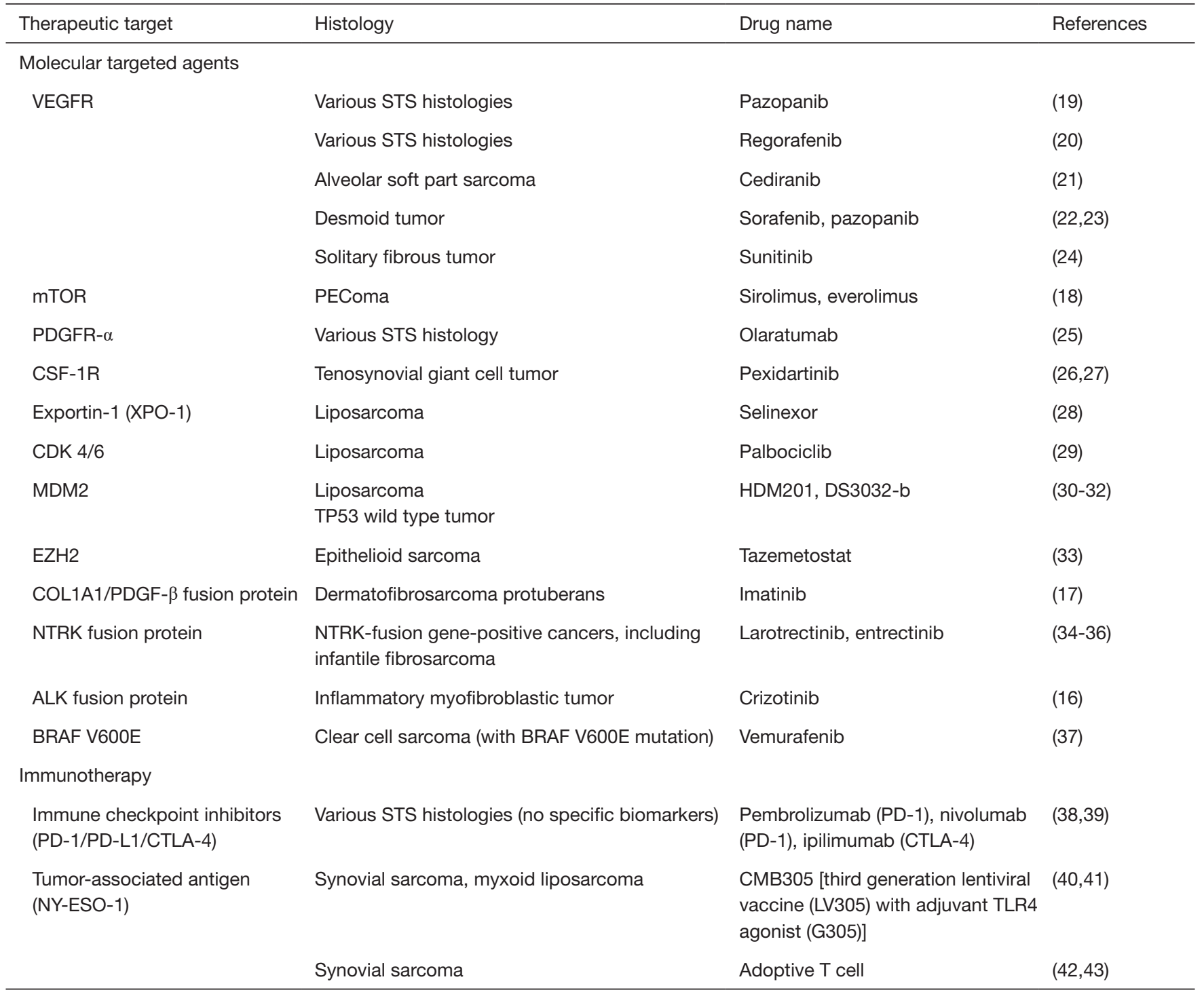

CDK, cyclin-dependent kinase; CSF-1R, colony stimulating factor-1 receptor; CTLA-4, cytotoxic T lymphocyte associated antigen-4; $\mathrm{EZH}$ 2, enhancer of zeste homolog 2; MDM2, murine double minute-2; mTOR, mammalian target of rapamycin; NTRK, neurotrophic tyrosine kinase; receptorPD-1/PD-L1, programmed cell death-1 (ligand); PDGFR- $\alpha$, platelet-derived growth factor receptor-alpha; PEComa, perivascular epithelioid cell tumor; STS, soft tissue sarcoma; VEGFR, vascular endothelial growth factor receptor.

activation. Its anti-tumor activity in combination with doxorubicin has been confirmed in preclinical cancer models. Also it is generally believed that it may exert activity in modifying microenvironment (62).

In a phase Ib/II trial for olaratumab, patients were randomly assigned to receive either olaratumab plus doxorubicin or doxorubicin alone. After completion of 8 cycles of doxorubicin, patients in the olaratumab plus doxorubicin group could receive olaratumab monotherapy until disease progression, and patients in the doxorubicin group were under observation and could select to receive olaratumab monotherapy after documented disease progression. In this study, although median PFS was improved by only 2.5 months [median PFS by investigators 6.6 vs. 4.1 months, hazard ratio (HR) 0.67, $\mathrm{P}=0.0615]$, overall survival (OS) was markedly extended by nearly 12 months with the combination (median OS $26.5 \mathrm{vs}$. 14.77 months, HR 0.46, $\mathrm{P}=0.003$ ) (25). The OS survival 
benefit were similar across subgroup analyses, including different histologies. Grade 3 or 4 neutropenia occurred more commonly in the combination arm but the rate of febrile neutropenia was similar between treatment arms (25). Although OS was the secondary endpoint in the study, the significant increase in OS led to the approval of the combination of doxorubicin and olaratumab in the treatment of advanced STS by both the US Food and Drug Administration (FDA) and European Medical Agency (EMA). A confirmatory randomized phase III trial of the combination of doxorubicin plus olaratumab versus single agent doxorubicin is underway. The recruitment has completed and the OS result is highly anticipated.

\section{Colony stimulating factor-1 receptor (CSF-1R) inbibitors}

Tenosynovial giant-cell tumors (TGCTs), also known as pigmented villonodular synovitis, is a locally invasive tumors of the joint or tendon sheath and characterized by proliferation of synoviocytes with infiltration of inflammatory cells including histiocytes and hemosiderinladen macrophages (63). Surgical resection is the main therapeutic modality. However, for those tumors with diffuse joint involvement, destructive surgery including amputation may be necessary (64). Most of TGCTs harbored a unique $t(1 ; 2)$ translocation generating a fusion gene that links the CSF1 gene on chromosome $1 \mathrm{p} 13$ to the COL6A3 gene on chromosome 2q35 $(65,66)$. Inhibition of signaling between CSF1 and CSF1 receptor (CSF1R) thus targets the underlying cause of the disease. CSF1 pathway is also associated with tumor-associated macrophages, which is also currently tested either single or in combination with other immunotherapies in solid tumors (67).

Pexidartinib (PLX3397) is a potent, selective CSF1R inhibitor that traps the kinase in an autoinhibited conformation (26). In a single-arm phase 2 study, among 23 TGCT patients, 12 patients had a partial response and 7 patients had stable disease. Responses usually occurred within the first 4 months of treatment, and the median duration of response exceeded 8 months (26). The most common adverse event is liver toxicity but most are reversible after discontinuation of the drug. In the phase III randomized study of pexidartinib versus placebo in TCGT (ENLIVEN), pexidartinib was started at $1,000 \mathrm{mg}$ per day in split dose and then decreased to $800 \mathrm{mg}$ per day in split dose because of concerns of liver toxicity. Tumor volume response was also a secondary endpoint in addition to standard RECIST criteria to compensate the difficulty in measuring the commonly irregular TGCT. The ORR per RECIST and volumetric criteria of pexidartinib $v s$. placebo were $39 \%$ vs. $0 \%$ and $56 \%$ vs. $0 \%$, respectively, suggesting that using a volume-based measurement more properly detected the efficacy of treatment in TGCT. Tumor response were similar when patients in the placebo arm were crossed to the pexidartinib arm after tumor progression (27). Eight patients discontinued pexidartinib because of hepatic adverse events, and 4 cases were serious nonfatal adverse events with increased bilirubin, one lasting around 7 months (27). Outside of the TGCT treatment program, liver toxicity is also observed. Although mostly are reversible, one patient did receive liver transplantation after the treatment of pexidartinib plus paclitaxel (27). Other agents such as imatinib with CSF1R inhibition activities also have been showed efficacy in treating TGCTs $(68,69)$. Overall, CSF1R inhibition is a reasonable choice with evident efficacy in TGCT. Although not common and mostly reversible, liver toxicities should receive greater attention when prescribing CSF1R inhibitors.

\section{Selective inbibitors of nuclear export (SINE)}

Exportin 1 (XPO1) is a critical mediator of nuclear export responsible for shuttling more than 200 known cargo proteins from the nucleus to the cytoplasm, including many tumor suppressor proteins (TSPs) (70). Selinexor, a novel SINE, could inhibit XPO1 by covalently and reversibly binding cysteine-528, an essential residue for XPO1 cargo binding. Inhibition of XPO1 results in nuclear accumulation of TSPs such as p53, pRb, p21, p27, and restores cell-cycle checkpoints and induces growth arrest and apoptosis in malignant cells (71).

XPO1 overexpression has been reported in several types of tumors and is correlated with poor prognosis (72-74). In the preclinical study, selinexor has demonstrated a universal response, both in vitro or in vivo, in a variety of sarcoma cell lines, including GISTs, LPS, LMS, ASPS and undifferentiated sarcoma (75). Unfortunately, in a phase Ib study, among 52 patients evaluable for response, none experienced an objective response, with only 17 (33\%) experiencing stable disease (SD) for 4 months or longer. However, it is interesting to find that for the 15 dedifferentiated liposarcoma (DD LPS) patients, six (40\%) of them showed a reduction in target lesion size from baseline and seven (47\%) of them showing SD for 4 months or longer (28). A registration-targeted clinical trial of selinexor specifically focused on in LPS is currently being 
investigated (ClinicalTrials.gov identifier: NCT 02606461).

\section{Cyclin-dependent kinase 4/6 (CDK4/6) inbibitors}

LPS are malignant mesenchymal tumors that are classified into three main biologic groups: well-differentiated (WD LPS) and dedifferentiated liposarcoma (DD LPS), myxoid/ round-cell LPS, and pleiomorphic LPS (76). WDLPS/ DDLPS are considered two sides of the spectrum of the same disease. The dedifferentiated component, which can be rapidly growing, aggressive, and metastatic, is considered to arise from the well-differentiated component, which can grow slowly (77). Both WDLPS and DDLPS are relatively resistant to chemotherapy, and few viable treatments exist for patients with locally advanced or metastatic disease (78).

The most common cytogenetic abnormalities found in WDLPS and DDLPS are supernumerary rings and giant chromosomes, which frequently contain amplifications in the long arm of chromosome 12 (12q13-q15) (79). Further studies revealed that this region contained oncogene cyclindependent kinase 4 (CDK4) and murine double minute-2 (MDM2) and found amplified in more than $90 \%$ of WDLPS/DDLPS (80). Gene profiling studies shown that CDK4 expression is 10 times higher in WDLPS/DDLPS than in normal fat tissue (81). In vitro study showed that inhibition of CDK4 expression with short hairpin RNA inhibits growth of WDLPS/DDLPS cells (82).

Palbociclib is a potent oral inhibitor of CDK4 and CDK6 that prevents downstream phosphorylation of the retinoblastoma protein (83). It has been demonstrated its efficacy in preclinical model of CDK4-amplified LPS cell lines and xenograft models (82). In a phase I study of palbociclib, two patients with Rb-positive WD or DDLPS achieved a long-term stable disease lasting several years (84). In a phase 2 study that enrolled 60 patients with advanced WD or DDLPS, treatment with palbociclib achieved a 57.2\% PFS at 12 weeks, and the median PFS was 17.9 weeks. There was 1 complete response. This agent showed promising result in the treatment of WD/DDLPS (29). Other CDK4/6 inhibitors including ribociclib and abemaciclib are also under investigation for WD/DD LPS patients.

\section{MDM2 inbibitors}

The tumor suppressor gene TP53, considered as "the guardian of the genome" is commonly mutated in around $50 \%$ of all cancer types. Another mechanism that could dysregulate $\mathrm{p} 53$ protein function is increasing the degradation of $\mathrm{p} 53$ through ubiquitination. MDM2 is responsible for $\mathrm{p} 53$ ubiquitination and is found to be overexpressed in certain types of cancer including sarcoma (20\%) (85). Besides WD and DD LPS, other sarcoma subtypes that are associated with $M D M 2$ amplification include intimal sarcoma and parosteal osteosarcoma $(86,87)$; however, other rare histologies such as rhabdomyosarcoma have also been reported to have MDM2 amplification occasionally (88). Single agent $\mathrm{mdm} 2$ inhibitors have shown some signals of activity in wild-type p 53 sarcoma patients, but mostly are WD or DD LPS with stable disease $(30,31)$. In addition, because MDM2 and CDK4 are commonly co-amplified in WD/DD LPS, a combination of $\mathrm{mdm} 2$ inhibitor HDM201 and CDK4/6 inhibitor ribociclib was also tested in this population that showed a $4 \%$ response rate and $49 \%$ stable disease rate (32). However, because stabilizing p53 through $\mathrm{mdm} 2$ inhibition causes cell apoptosis, the common class effect toxicity for $\mathrm{mdm} 2$ inhibitors is bone marrow toxicities. Grade 3 or 4 neutropenia or even prolonged thrombocytopenia has been experienced by $30 \%$ or higher of patients (30-32). Multiple different drugs dosage schedules are currently explored to find the best schedule. For mdm2 inhibitors, although some efficacy potential is noted in STS with $M D M 2$ amplification or a wild type $T P 53$, reaching a balance between the efficacy and toxicities will be critical to the drug development process of $\mathrm{mdm} 2$ inhibitors.

\section{Epigenetic modifying drugs}

Histone deacetylase inhibitors, although promising in preclinical studies of sarcoma, did not show activity in unselected sarcoma patients (89). However, newer generation drugs that target the chromatin modifying machinery enhancer of zeste homolog 2 (EZH2) have shown promising results. EZH2 is a major catalytic unit in the polycomb repressive complex 2 (PRC2) that is commonly aberrantly expressed in tumors (90). Another chromatin modifying complex in the cell, the SWI/SNF, acts as a suppressor of EZH2. When a key component of SWI/SNF complex, SMARCB1 (SWI/SNF related matrixassociated actin-dependent regulator of chromatin subfamily B member 1, or more commonly INI-1) lost its function, tumor cells are more likely to shift the balance toward EZH2 reprogramming, which more favors progression (91). INI-1 is commonly lost in epithelioid sarcoma, synovial sarcoma, epithelioid malignant peripheral nerve sheath tumor, myoepithelial tumor, and extraskeletal myxoid 
chondrosarcoma $(92,93)$. It is noteworthy that functional loss of INI-1 is observed in more than $90 \%$ of epithelioid sarcoma patients (94). An EZH2 small molecular inhibitor, tazemetostat, is currently being tested in a multiple arms two-step phase II study including rhabdoid tumors, synovial sarcoma, INI-1 loss malignancy, epithelioid sarcoma and poorly-differentiated chordoma. Confirmed response were seen in epithelioid sarcoma, with the ORR $13 \%$ among 31 patients and a median PFS of 5.7 months (33). However, in the synovial sarcoma cohort, no responders were noted (95). The adverse events were generally well tolerated. The efficacy signal of epithelioid sarcoma warranted an expansion of the ES patient for further testing (ClinicalTrials.gov identifier: NCT02601950).

\section{Fusion-gene targets}

Specific fusions are pathognomonic and driver for many STS histologies and have been main targets of therapeutic potential. The success of crizotinib in $A L K$ translocation positive IMT (16) and imatinib in COL1A1-PDGFB positive DFSP (17) certainly speaks that it is worthwhile to develop drugs specific for fusion-genes.

The tropomyosin receptor kinase (TRK) plays significant role in neuronal growth and differentiation but the fusion of the coding gene NTRK with a non-related gene have been found in infrequently in various adult and pediatric solid tumors with transformative capability (96). Most often, the 5'-end of the NTRK-1, -2 or -3 gene that includes the kinase domain of TRK protein is fused in-frame with the 3 '-end of another gene, causing an oncogenic fusion protein (96). Although rarely found, certain STS subtypes such as infantile fibrosarcoma and uterine sarcoma may have higher chances of harboring NTRK-specific fusion genes $(34,97)$. The first report of the efficacy of TRK kinase inhibitor was reported in a sarcoma patient with $L M N A$ TRKA fusion oncoprotein (35). The tumors responded exceptionally well to the single agent larotrectinib, a panTRK inhibitor. In further phase I/II study of larotrectinib, an ORR of $78 \%$ and a complete response (CR) rate of $13 \%$ were observed among adult and pediatric patients who harbored NTRK fusions, including 10 of the 11 patients with sarcoma (34). Another pan-TRK, ROS, and ALK inhibitor, entrectinib, also demonstrated promising clinical activity in patients harboring NTRK fusion gene, including patients with brain metastases (36). This result further highlights the need to screen multiple possible molecular targets in STS patients in a tissue agnostic fashion in order to find the best way to treatment the tumor.

\section{Precision oncology and STS treatment}

The uprising of inexpensive and efficient massively parallel next generation sequencing (NGS) has changed the clinical practice of oncology (98). Patients can now obtain the genetic alterations landscape for mutations, indels, amplifications and translocations of hundreds of actionable targets in one test (99). Institutional studies have shown that NGS testing may increase the chance of finding a molecular-based treatment in rare cancers such as sarcoma. In a retrospective analysis from the Memorial Sloan Kettering Cancer Center, 5,635 sarcoma patients worldwide were screened for a NGS panel that included 405 cancer-related genes and 265 genes rearranged in RNA. By using an in-house bioinformatics pipeline OncoKB (100), they found that $16 \%$ and $7 \%$ of the patients had treatmentlinked alterations known to respond to FDA approved or study drug (101). Another sarcoma-specific NGS database analysis showed that of 584 sarcoma patients, at least one targetable genetic alteration was noted in $41 \%$ of patients (102).

Despite the promising initial findings, some caveats exist. First, many of the "actionable" alterations selected into the NGS panels are based on preclinical or sound biological speculation but not by evidence of clinical efficacy. For instance, the common actionable mutations detected in STS included TP53, ATRX, MDM2, CDK4, and RB (103). Except that $M D M 2$ and $C D K 4$ have specific inhibitors, the loss of function of tumor suppressor genes such as TP53, $A T R X$ and $R B$ are much more difficult to find a target to treat. Even with MDM2 and CDK4/6 inhibitors, the clinical trial results of these drugs in STS are not exuberant (as mentioned in previous section). Secondly, it is still uncertain if actionable alterations are equal among different cancer types. For instance, melanoma with BRAF V600E mutations responded exceptionally well to BRAF inhibitor vemurafenib (104). Based the concept that BRAF mutation may be the driver oncogene in all cancer types, a basket clinical trial for nonmelanoma patients was initiated. In this study, lung cancer, Erdheim-Chester disease/Langerhans'cell histiocytosis, and clear cell sarcoma patients with $B R A F$ V600E mutation responded well to vemurafenib (37). However, colorectal cancer patients responded poorly (37). The discrepant responses to driver mutations in different cancer types also brought skeptical viewpoints toward the actual benefit of NGS testing in daily clinical setting (105). 
To provide a framework for precision oncology in across all cancer types, both the National Cancer Institution (NCI) and American Society of Clinical Oncology (ASCO) have initiated precision oncology clinical trials: the NCIMolecular Analysis for Therapy Choice (MATCH) and ASCO Targeted Agent and Profiling Utilization Registry (TAPUR) (106). Both clinical trials have been well accepted by both the medical community and the patients, hence with a higher than expected recruitment rate, especially for rare and uncommon cancers like sarcoma. In NCIMATCH, about $60 \%$ of the enrolled patients are cancers other than colon, rectal, breast, non-small cell lung, and prostate, much exceeding the previous expected rate of $25 \%$ (107). Enrolled patients will be allocated to specific molecular treatment groups by their genomic sequencing results and to monitor the tumor response (108). Pre-set rule-based criteria will be used to determine the efficacy of these molecularly-matched agents. The results of these precision oncology clinical trials are highly anticipated and may bring more genetically or molecularly-tailored precision medicine into the treatment paradigm of advanced STS patients.

\section{Immunotherapy in STS}

The immune system plays a significant role in control tumorigenesis (44). One of the mechanisms that the cancer cells utilize to evade the immune system is to exploit the immunosuppressive molecules that are physiologically substantial to avoid autoimmune and uncontrolled immune responses-the immune checkpoint signaling pathways that include cytotoxic T-lymphocyte associated protein-4 (CTLA-4) and the programmed cell death receptor-1 (PD-1) and its ligand, PD-L1 (109). The success of immune checkpoint inhibitor (ICI) monoclonal antibodies such as pembrolizumab (anti-PD-1), nivolumab (anti-PD-1), atezolizumab (anti-PD-L1), avelumab (anti-PD-L1), durvalumab (anti-PD-L1), and ipilimumab (anti-CTLA-4) in melanoma, lung cancer, renal cell carcinoma and other malignancies have resulted in a paradigm shift in the cancer therapy field (109).

Both soft tissue and bone sarcoma have evidence suggesting that the immune system is involved in sarcomagenesis. Dr. Coley first noted immune-induced tumor response in sarcoma patients after erysipelas infection more than 130 years ago (110). Many sarcoma subtypes such as LMS, angiosarcoma, and Kaposi's sarcoma are more common among patients who are immunosuppressive such as allograft transplantation receivers and HIV-infected patients $(111,112)$. Furthermore, the amount and type of tumor infiltrating lymphocytes (TILs) have also been documented to correlate with prognosis in both STS and bone sarcomas $(113,114)$. Tumor associated antigens (TAAs), which may lead to tumor antigen expression and priming of the immune system, are also common in STS (discussed in the next section). Taken together, these evidences support that immune system is substantial in STS and provide the rationale to investigate immunotherapy in advanced STS.

\section{Immune checkpoint inbibitors (ICI) in sarcoma}

SARC028 is one of the largest prospective clinical trial to date to test the efficacy of single agent ICI in bone and STS (38). In SARC 028, 40 STS patients [10 LMS, 10 LPS, 10 synovial sarcoma (SS), and 10 undifferentiated pleomorphic sarcoma (UPS)] were treated with single agent pembrolizumab 200mg every 3 weeks. All patients have received at least one-line of prior therapy. The results showed an overall objective response rate (ORR) of $18 \%$ in 40 STS patients and a median duration of response of 33 weeks (38). However, ORR among histologies varied. The histology that had the highest ORR was UPS, with 4 out of $10(40 \%)$ patients having tumor response; LMS had the lowest ORR with 0 out of $10(0 \%)$ of patients responded. Two patients with DDLPS and 1 patient of SS also had tumor response (38). The patient numbers are too small to reach final conclusion and expansion studies of pembrolizumab in DD LPS and UPS is ongoing. Interestingly, another study using nivolumab $(3 \mathrm{mg} / \mathrm{kg}$ every 2 weeks) in uterine LMS also saw 0 responders out of 12 patients (115). The study was closed prematurely with the lack of efficacy. Although it is too early to specify that certain histologies of STS are unlikely to response to ICI, different histologies are likely to have different immune evasion mechanisms. For instance, studies have suggested that loss of PTEN protein in LMS may be associated with ICI treatment resistance (116).

The combination of nivolumab and ipilimumab have the advantage to eliminate two inhibitory signals on $\mathrm{T}$ cells and potentially improve the response rate in solid tumors (109). In melanoma and renal cell carcinoma, the combination of nivolumab and ipilimumab have shown to improve response rate and disease control over either single agent nivolumab or ipilimumab (in melanoma) (117) or single agent sunitinib (in intermediate or poor-risk renal cell carcinoma patients) (118). But the toxicities because of immune- 
related adverse events were higher in the combination arm as well, with more than $40 \%$ of patients had grade 3 or higher toxicities $(117,118)$. The combination of nivolumab and ipilimumab was also tested in the Sarcoma Alliance Study A091401. The study was designed as a two-arm study to either single agent nivolumab or the combination of nivolumab plus ipilimumab. Patients who progressed on the nivolumab arm could choose to cross-over to the combination of nivolumab and ipilimumab (39). The ORR for single agent nivolumab and the combination was $5 \%$ ( 2 of 38 ) and 16\% (6 of 38). Median PFS and OS for nivolumab and nivolumab plus ipilimumab combination was 1.7 and $10.7,4.1$ and 14.3 months, respectively. A lower dose of ipilimumab $(1 \mathrm{mg} / \mathrm{kg}$ as compared to $3 \mathrm{mg} / \mathrm{kg}$ in other studies) led to a lower than expected grade 3 or higher treatment related adverse events (14\%) in the combination arm (39). In the Alliance study, they also observed that STS subtypes such as LMS, angiosarcoma, myxofibrosarcoma, and ASPS responded to ICI treatment. However, not all combination of immunotherapies showed positive results. Metronomic cyclophosphamide is a lowdose daily administrative regimen that could decrease the number of regulatory $\mathrm{T}$ cells (Tregs) in the peripheral blood and could antagonize the immunosuppressive microenvironment (119). In a study to evaluate the combination of metronomic cyclophosphamide plus pembrolizumab, out of 50 evaluable patients, only one solitary fibrous tumor patients responded to the combination and the 6-month PFS rate for LMS $(n=15)$ and UPS $(n=16)$ were both $0 \%(120)$. This result further signifies that a better understanding of the predictive factors that is associated with response to immunotherapy is warranted.

\section{Modulation of the tumor microenvironment to improve response to immunotherapy}

The tumor microenvironment plays an important role in determining the response to immunotherapy (121) and STS are generally "cold tumors" with less T cell infiltration (122). Angiogenesis molecules are also involved in dendritic cell maturation and $\mathrm{T}$ cell trafficking and are generally immunosuppressive in tumor immune system (123). In two studies, the administration of anti-VEGF antibody before atezolizumab or ipilimumab showed increased $\mathrm{T}$ cell infiltration in the tumor microenvironment $(124,125)$. In a phase Ib study, axitinib (an VEGFR 1, 2 and, 3 inhibitor) plus pembrolizumab combination in renal cell carcinoma yielded an $73 \%$ (38 out of 52) ORR (126). Axitinib and pembrolizumab combination was also tested in STS patients. In 30 evaluable patients that included ASPS, UPS, LMS, and other histologies, the overall ORR was $21.9 \%$. Interestingly, ASPS histology have the highest ORR at $45 \%$ (127). Other studies also have suggested that ASPS may be more susceptible to ICI because of a genomic mismatch repair deficiency mutation signature (128), which is associated with a higher response to ICI in other cancer types (129). It is now widely accepted that molecular targeted agents not only affect the cancer cell but also can influence or reshape the contexture of the tumor immune microenvironment (123). Clinical trials that integrate biomarker study to fully understand the modulation of the immune microenvironment in the combination of anti-angiogenic molecules or other targeted agents with immunotherapy are highly anticipated.

\section{Expression of PD-L1 as predictive biomarkers of response to ICI}

Expression of PD-L1 on tumor cells may be a reflection of immune evasion through the PD-1/PD-L1 axis and more likely to be associated with increased $\mathrm{T}$ cell infiltration (121). In many solid tumors, increased expression of PD-L1 on tumor cells are correlated with ICI treatment benefit (109). The expression of PD-L1 on sarcoma tumor cells varies with histology but UPS generally have the highest PD-L1 expression $(122,130)$. In SARC 028 , although only 2 patients ( $5 \%$ ) had positive PD-L1 expression on tumor cells, both of these patients are UPS and were responsive to pembrolizumab (38). Interestingly, high PD-L1 expression on the immune cells (including both lymphocytes and macrophages) of STS have been found in many studies $(120,122,130)$. Recent biomarkers studies have also suggested that a combined analyses of PD-L1 on both tumor cells and immune cells may be more predictive of the benefit to ICI than tumor cells alone $(131,132)$. Further studies to delineate the role of PD-L1 on both tumor cells and immune cells in STS and correlation with ICI treatment benefit is further warranted.

\section{Targeting tumor-associated antigens (TAA)}

Tumor possess antigens that are not commonly observed or low quantity in normal cells. Generally, TAA are categorized in 3 types: antigens that are uniquely only expressed on tumor cells (e.g., neoantigens), antigens 
that have much higher quantity as compared with normal cells (e.g., epidermal growth factor receptor), or antigens that are only expressed on tumor cells and normal body parts that are immune-neglect [e.g., cancer-testis antigen (CTA)] (133). CTA has been an attractive target for immunotherapy because of the lack of human leukocyte antigen (HLA)-class I molecule on male germ cells, limiting $\mathrm{T}$ cell responses that may come along after antigen stimulation (134).

NY-ESO-1 is a TAA that has been found to be expressed in testicular and ovarian fetal germ cells and placenta. As in STS, the common histologies with NY-ESO-1 expression are synovial sarcoma (SS) and myxoid round cell liposarcoma (MCL LPS). In a series of 25 MCL LPS patients, NY-ESO-1 was found to be homogeneously expressed in $70 \%$ of patients and both the myxoid and round cell component tend to stain positive for NYESO-1 (135). Around $50-80 \%$ of SS are positive for NYESO-1. Similar to the findings of MRC LPS, either monophasic or biphasic (both the epithelial and spindle cell components) staining equally positive for NY-ESO-1 $(136,137)$. Other sarcomas that has a lower percentage of NY-ESO-1 expression included myxofibrosarcoma (35\%) and conventional chondrosarcoma (28\%) (136). Overall, because of the high and homogenous expression in certain types of STS and the limited expression in normal tissue, NY-ESO-1 has been an attractive target for immunotherapy in STS.

Recent advancement in targeting NY-ESO-1 generally focuses on two methods to harness the immune system to target NY-ESO-1 positive STS: (I) using a lentiviral vector with NY-ESO-1 peptide to stimulate the immune system; (II) using adoptive $\mathrm{T}$ cells with $\mathrm{T}$ cell-receptor (TCR) targeting NY-ESO-1.

\section{Viral cancer vaccine to active the immune system toward NY-ESO-1}

Dendritic cells (DC) play an important role in priming and activating the $\mathrm{T}$ cells against cancer cells. Through the antigen-presentation machinery in the cytoplasm, the DC could efficiently load the exogenous peptide into the MHC-complex to generate T cell responses (138). Viral vaccines with $\mathrm{DC}$ tropism have shown some anti-tumor efficacy either through ex vivo stimulation or direct tumor injection (139). LV305 is a vaccine with a third generation lentiviral vector that utilizes a modified Sindbis virus coat with tropism toward dendritic cells. LV305 loaded with the NY-ESO-1 peptide injected subcutaneously have been shown to be safe (140). In a phase I trial testing LV305 in NY-ESO-1+ tumors, a patient with SS had a PR $(80 \%$ regression) with evidence of increased tumor-specific $\mathrm{T}$ cell clones (40). Furthermore, a primed-boost regimen (CMB305) was developed to enhance the immune response. CMB305 is composed of LV305 and an adjuvant G305 that contains a full-length NY-ESO-1 protein and a tolllike receptor 4 agonist (140). CMB305 regimen schedule is to start with the LV305 priming vaccines on Days 0, 21, 49 , and 77 while the adjuvant G305 starts on D35 every 4 weeks for 3 doses then every 8 weeks for up to one year. In the phase I trial that included 25 advanced sarcoma patients (80\% with homogeneous NY-ESO-1 expression), 1-year survival rate higher than $85 \%$ was observed in SS and MRC LPS patients. Immunity against NY-ESO-1 was induced in at least $60 \%$ of SS or myxoid round cell LPS patients (41). A randomized phase III study investigating the role of CMB305 in advanced SS positive for NY-ESO-1 in the maintenance setting after first-line chemotherapy is currently undergoing (ClinicalTrials.gov Identifier: NCT03520959).

\section{NY-ESO-1-specific adoptive T cells}

The first generation of adoptive $\mathrm{T}$ cell therapy involves the extraction of TILs and re-infused into the patients after ex vivo stimulation (141). Albeit promising activity, the difficulty in obtaining of TILs makes this method less attractive. The second generation of adoptive $\mathrm{T}$ cells involves a transfection of retroviral vector with a genetically modified T-cell receptor that could recognize NY-ESO-1. In the initial study, Dr. Rosenberg and colleagues at the NCI tested the adoptive TCR in SS and melanoma patients (42). Patients need to receive pre-conditioning lympho-depletion chemotherapy regimens such as fludarabine and cyclophosphamide and interleukin-2 injections along with engineered $\mathrm{T}$ cells to boost the proliferation. In the first 6 patients with SS, all with HLA-A ${ }^{*} 0201$ haplotype and high expression of NY-ESO-1, four had objective response lasting 5-18 months. Toxicities were generally considered from the lympho-depleting chemotherapy or interleukin-2 and not from the injected adoptive T cells (42). Because of the promising activity, an expansion cohort including 12 SS patients was initiated. Among these patients, 5 received addition NY-ESO-1 peptide vaccine to boost the anti-tumor immunity (43). With 7 patients in the expansion cohort having objective 
response, the overall response rate of NY-ESO-1 TCR in SS was $61 \%$, with response lasting from 3 to $47+$ months. However, re-treatment with NY-ESO-1 TCR in recurrent patient only showed short or no response. The 3 - and 5 -year OS was $38 \%$ and $14 \%$; and 4-year PFS was at $11 \%$. Interestingly, the addition of NY-ESO-1 vaccination was not correlated with an increased response rate but the number of infused T cells and NY-ESO-1-reactive T cells are more likely predictors of response (43).

There are still some limitations of the adoptive $\mathrm{T}$ cell therapy in SS or other sarcoma with strong expression of NY-ESO-1. The strong preconditioning chemotherapy regimen and interleukin-2 injection excludes patients that are more fragile from receiving T-cell infusion. Studies that are testing different but lower intensity of chemotherapy before NY-ESO-1 TCR infusion are being investigated. Secondly, currently NY-ESO-1 TCR are generated with the specificity to HLA-A2 haplotype. Although HLA-A2 may be the most common haplotype in Western Countries (27\% of US Caucasians), other ethnicities will have a different HLA-A landscape pattern that may limit the application of the NY-ESO-1 TCR (142). Further understanding the NY-ESO-1 presentation to different haplotypes of HLA may improve the scalability and applicability to a wider population or ethnicity of STS patients with NY-ESO-1 expression.

\section{Summary}

Two trends are pushing the frontiers in the treatment of oncology patients: molecular target agents that more closely matched to the genomic alterations or biological mechanisms of the cancer and reactivation or modulation of the immune system to fight cancer. STS treatments have not lagged too much behind in both of these two categories. In molecular targeted agents, many anti-angiogenic factors have shown activity in STS and PDGFRA monoclonal antibody in combination with doxorubicin have also improved the survival of advanced STS patients. Other STS-specific genomic or molecular target inhibitors such as CSF-1R, SINE, MDM2, CDK4, and EZH2 are also showing promising future. How to match the current wave of precision oncology with sound and solid clinical trial evidence is also a key to finding the optimal treatments of each STS patient.

In immunotherapy treatments, single agent ICI may have activity in certain histologies but definitely is not the answer for most advanced STS patients. Combinations of either two ICI inhibitors or other molecular targeted agents that can modulate the tumor microenvironment is under intense investigation. Other immunotherapy methods such as viral vaccines or adoptive $\mathrm{T}$ cells are front-runners that target the CTA NY-ESO-1. Immune system is like the yin and yang, always in process of balancing the enhancing and suppressive factors of the immune system. A deeper understanding of the immune contexture to understand the resistance in immunotherapy plays another key role to extending the envelope of the frontier in the treatment of advanced STS.

\section{Acknowledgements}

None.

\section{Footnote}

Conflicts of Interest: The authors have no conflicts of interest to declare.

\section{References}

1. Jemal A, Siegel R, Xu J, et al. Cancer statistics, 2010. CA Cancer J Clin 2010;60:277-300.

2. Pervaiz N, Colterjohn N, Farrokhyar F, et al. A systematic meta-analysis of randomized controlled trials of adjuvant chemotherapy for localized resectable soft-tissue sarcoma. Cancer 2008;113:573-81.

3. Beane JD, Yang JC, White D, et al. Efficacy of adjuvant radiation therapy in the treatment of soft tissue sarcoma of the extremity: 20-year follow-up of a randomized prospective trial. Ann Surg Oncol 2014;21:2484-9.

4. Lahat G, Tuvin D, Wei C, et al. New perspectives for staging and prognosis in soft tissue sarcoma. Ann Surg Oncol 2008;15:2739-48.

5. Liu CY, Yen CC, Chen WM, et al. Soft tissue sarcoma of extremities: the prognostic significance of adequate surgical margins in primary operation and reoperation after recurrence. Ann Surg Oncol 2010;17:2102-11.

6. Anaya DA, Lahat G, Liu J, et al. Multifocality in retroperitoneal sarcoma: a prognostic factor critical to surgical decision-making. Ann Surg 2009;249:137-42.

7. Nussbaum DP, Rushing CN, Lane WO, et al. Preoperative or postoperative radiotherapy versus surgery alone for retroperitoneal sarcoma: a case-control, propensity score-matched analysis of a nationwide clinical oncology 
database. Lancet Oncol 2016;17:966-75.

8. Judson I, Verweij J, Gelderblom H, et al. Doxorubicin alone versus intensified doxorubicin plus ifosfamide for first-line treatment of advanced or metastatic soft-tissue sarcoma: a randomised controlled phase 3 trial. Lancet Oncol 2014;15:415-23.

9. Seddon B, Strauss SJ, Whelan J, et al. Gemcitabine and docetaxel versus doxorubicin as first-line treatment in previously untreated advanced unresectable or metastatic soft-tissue sarcomas (GeDDiS): a randomised controlled phase 3 trial. Lancet Oncol 2017;18:1397-410.

10. Schöffski P, Chawla S, Maki RG, et al. Eribulin versus dacarbazine in previously treated patients with advanced liposarcoma or leiomyosarcoma: a randomised, open-label, multicentre, phase 3 trial. Lancet 2016;387:1629-37.

11. Demetri GD, von Mehren M, Jones RL, et al. Efficacy and Safety of Trabectedin or Dacarbazine for Metastatic Liposarcoma or Leiomyosarcoma After Failure of Conventional Chemotherapy: Results of a Phase III Randomized Multicenter Clinical Trial. J Clin Oncol 2016;34:786-93.

12. Demetri GD, Reichardt P, Kang YK, et al. Efficacy and safety of regorafenib for advanced gastrointestinal stromal tumours after failure of imatinib and sunitinib (GRID): an international, multicentre, randomised, placebocontrolled, phase 3 trial. Lancet 2013;381:295-302.

13. Demetri GD, van Oosterom AT, Garrett CR, et al. Efficacy and safety of sunitinib in patients with advanced gastrointestinal stromal tumour after failure of imatinib: a randomised controlled trial. Lancet 2006;368:1329-38.

14. Blanke CD, Demetri GD, von Mehren M, et al. Longterm results from a randomized phase II trial of standardversus higher-dose imatinib mesylate for patients with unresectable or metastatic gastrointestinal stromal tumors expressing KIT. J Clin Oncol 2008;26:620-5.

15. Lovly CM, Gupta A, Lipson D, et al. Inflammatory myofibroblastic tumors harbor multiple potentially actionable kinase fusions. Cancer Discov 2014;4:889-95.

16. Butrynski JE, D'Adamo DR, Hornick JL, et al. Crizotinib in ALK-rearranged inflammatory myofibroblastic tumor. N Engl J Med 2010;363:1727-33.

17. McArthur GA, Demetri GD, van Oosterom A, et al. Molecular and clinical analysis of locally advanced dermatofibrosarcoma protuberans treated with imatinib: Imatinib Target Exploration Consortium Study B2225. J Clin Oncol 2005;23:866-73.

18. Wagner AJ, Malinowska-Kolodziej I, Morgan JA, et al. Clinical activity of mTOR inhibition with sirolimus in malignant perivascular epithelioid cell tumors: targeting the pathogenic activation of mTORC1 in tumors. J Clin Oncol 2010;28:835-40.

19. Sleijfer S, Ray-Coquard I, Papai Z, et al. Pazopanib, a multikinase angiogenesis inhibitor, in patients with relapsed or refractory advanced soft tissue sarcoma: a phase II study from the European organisation for research and treatment of cancer-soft tissue and bone sarcoma group (EORTC study 62043). J Clin Oncol 2009;27:3126-32.

20. Mir O, Brodowicz T, Italiano A, et al. Safety and efficacy of regorafenib in patients with advanced soft tissue sarcoma (REGOSARC): a randomised, double-blind, placebocontrolled, phase 2 trial. Lancet Oncol 2016;17:1732-42.

21. Kummar S, Allen D, Monks A, et al. Cediranib for metastatic alveolar soft part sarcoma. J Clin Oncol 2013;31:2296-302.

22. Gounder MM, Lefkowitz RA, Keohan ML, et al. Activity of Sorafenib against desmoid tumor/deep fibromatosis. Clin Cancer Res 2011;17:4082-90.

23. Szucs Z, Messiou C, Wong HH, et al. Pazopanib, a promising option for the treatment of aggressive fibromatosis. Anticancer Drugs 2017;28:421-6.

24. Stacchiotti S, Negri T, Libertini M, et al. Sunitinib malate in solitary fibrous tumor (SFT). Ann Oncol 2012;23:3171-9.

25. Tap WD, Jones RL, Van Tine BA, et al. Olaratumab and doxorubicin versus doxorubicin alone for treatment of softtissue sarcoma: an open-label phase $1 \mathrm{~b}$ and randomised phase 2 trial. Lancet 2016;388:488-97.

26. Tap WD, Wainberg ZA, Anthony SP, et al. StructureGuided Blockade of CSF1R Kinase in Tenosynovial GiantCell Tumor. N Engl J Med 2015;373:428-37.

27. Tap WD, Gelderblom H, Stacchiotti S, et al. Final results of ENLIVEN: A global, double-blind, randomized, placebo-controlled, phase 3 study of pexidartinib in advanced tenosynovial giant cell tumor (TGCT). J Clin Oncol 2018;36:11502.

28. Gounder MM, Zer A, Tap WD, et al. Phase IB Study of Selinexor, a First-in-Class Inhibitor of Nuclear Export, in Patients With Advanced Refractory Bone or Soft Tissue Sarcoma. J Clin Oncol 2016;34:3166-74.

29. Dickson MA, Schwartz GK, Keohan ML, et al. Progression-Free Survival Among Patients With WellDifferentiated or Dedifferentiated Liposarcoma Treated With CDK4 Inhibitor Palbociclib: A Phase 2 Clinical Trial. JAMA Oncol 2016;2:937-40.

30. Bauer TM, Gounder MM, Weise AM, et al. A phase 1 study of MDM2 inhibitor DS-3032b in patients with well/ 
de-differentiated liposarcoma (WD/DD LPS), solid tumors (ST) and lymphomas (L). J Clin Oncol 2018;36:11514-.

31. Ray-Coquard I, Blay JY, Italiano A, et al. Effect of the MDM2 antagonist RG7112 on the P53 pathway in patients with MDM2-amplified, well-differentiated or dedifferentiated liposarcoma: an exploratory proof-ofmechanism study. Lancet Oncol 2012;13:1133-40.

32. Razak AA, Bauer S, Blay JY, et al. Abstract CT009: Results of a dose- and regimen-finding Phase Ib study of HDM201 in combination with ribociclib in patients with locally advanced or metastatic liposarcoma. Cancer Res 2018;78:CT009.

33. Gounder MM, Stacchiotti S, Schöffski P, et al. Phase 2 multicenter study of the EZH2 inhibitor tazemetostat in adults with INI1 negative epithelioid sarcoma (NCT02601950). J Clin Oncol 2017;35:11058-.

34. Drilon A, Laetsch TW, Kummar S, et al. Efficacy of Larotrectinib in TRK Fusion-Positive Cancers in Adults and Children. N Engl J Med 2018;378:731-9.

35. Doebele RC, Davis LE, Vaishnavi A, et al. An Oncogenic NTRK Fusion in a Patient with Soft-Tissue Sarcoma with Response to the Tropomyosin-Related Kinase Inhibitor LOXO-101. Cancer Discov 2015;5:1049-57.

36. Drilon A, Siena S, Ou SI, et al. Safety and Antitumor Activity of the Multitargeted Pan-TRK, ROS1, and ALK Inhibitor Entrectinib: Combined Results from Two Phase I Trials (ALKA-372-001 and STARTRK-1). Cancer Discov 2017;7:400-9.

37. Hyman DM, Puzanov I, Subbiah V, et al. Vemurafenib in Multiple Nonmelanoma Cancers with BRAF V600 Mutations. N Engl J Med 2015;373:726-36.

38. Tawbi HA, Burgess M, Bolejack V, et al. Pembrolizumab in advanced soft-tissue sarcoma and bone sarcoma (SARC028): a multicentre, two-cohort, single-arm, openlabel, phase 2 trial. Lancet Oncol 2017;18:1493-501.

39. D'Angelo SP, Mahoney MR, Van Tine BA, et al. Nivolumab with or without ipilimumab treatment for metastatic sarcoma (Alliance A091401): two open-label, non-comparative, randomised, phase 2 trials. Lancet Oncol 2018;19:416-26.

40. Pollack SM, Lu H, Gnjatic S, et al. First-in-Human Treatment With a Dendritic Cell-targeting Lentiviral Vector-expressing NY-ESO-1, LV305, Induces Deep, Durable Response in Refractory Metastatic Synovial Sarcoma Patient. J Immunother 2017;40:302-6.

41. Somaiah N, Chawla SP, Block MS, et al. Immune response, safety, and survival impact from CMB305 in NYESO-1+ recurrent soft tissue sarcomas (STS). J Clin Oncol
2017;35:11006.

42. Robbins PF, Morgan RA, Feldman SA, et al. Tumor regression in patients with metastatic synovial cell sarcoma and melanoma using genetically engineered lymphocytes reactive with NY-ESO-1. J Clin Oncol 2011;29:917-24.

43. Robbins PF, Kassim SH, Tran TL, et al. A pilot trial using lymphocytes genetically engineered with an NYESO-1-reactive T-cell receptor: long-term followup and correlates with response. Clin Cancer Res 2015;21:1019-27.

44. Hanahan D, Weinberg RA. Hallmarks of cancer: the next generation. Cell 2011;144:646-74.

45. Potti A, Ganti AK, Tendulkar K, et al. Determination of vascular endothelial growth factor (VEGF) overexpression in soft tissue sarcomas and the role of overexpression in leiomyosarcoma. J Cancer Res Clin Oncol 2004;130:52-6.

46. Pakos EE, Goussia AC, Tsekeris PG, et al. Expression of vascular endothelial growth factor and its receptor, KDR/Flk-1, in soft tissue sarcomas. Anticancer Res 2005;25:3591-6.

47. Chao C, Al-Saleem T, Brooks JJ, et al. Vascular endothelial growth factor and soft tissue sarcomas: tumor expression correlates with grade. Ann Surg Oncol 2001;8:260-7.

48. Yudoh K, Kanamori M, Ohmori K, et al. Concentration of vascular endothelial growth factor in the tumour tissue as a prognostic factor of soft tissue sarcomas. Br J Cancer 2001;84:1610-5.

49. Yoon SS, Segal NH, Olshen AB, et al. Circulating angiogenic factor levels correlate with extent of disease and risk of recurrence in patients with soft tissue sarcoma. Ann Oncol 2004;15:1261-6.

50. Hayes AJ, Mostyn-Jones A, Koban MU, et al. Serum vascular endothelial growth factor as a tumour marker in soft tissue sarcoma. Br J Surg 2004;91:242-7.

51. Schutz FA, Choueiri TK, Sternberg CN. Pazopanib: Clinical development of a potent anti-angiogenic drug. Crit Rev Oncol Hematol 2011;77:163-71.

52. Maki RG, D'Adamo DR, Keohan ML, et al. Phase II study of sorafenib in patients with metastatic or recurrent sarcomas. J Clin Oncol 2009;27:3133-40.

53. Lieberman PH, Brennan MF, Kimmel M, et al. Alveolar soft-part sarcoma. A clinico-pathologic study of half a century. Cancer 1989;63:1-13.

54. Portera CA Jr, Ho V, Patel SR, et al. Alveolar soft part sarcoma: clinical course and patterns of metastasis in 70 patients treated at a single institution. Cancer 2001;91:585-91.

55. Reichardt P, Lindner T, Pink D, et al. Chemotherapy 
in alveolar soft part sarcomas. What do we know? Eur J Cancer 2003;39:1511-6.

56. Ladanyi M, Lui MY, Antonescu CR, et al. The der(17) $\mathrm{t}(\mathrm{X} ; 17)(\mathrm{p} 11 ; \mathrm{q} 25)$ of human alveolar soft part sarcoma fuses the TFE3 transcription factor gene to ASPL, a novel gene at 17q25. Oncogene 2001;20:48-57.

57. Stockwin LH, Vistica DT, Kenney S, et al. Gene expression profiling of alveolar soft-part sarcoma (ASPS). BMC Cancer 2009;9:22.

58. Stacchiotti S, Negri T, Zaffaroni N, et al. Sunitinib in advanced alveolar soft part sarcoma: evidence of a direct antitumor effect. Ann Oncol 2011;22:1682-90.

59. Nakamura T, Matsumine A, Kawai A, et al. The clinical outcome of pazopanib treatment in Japanese patients with relapsed soft tissue sarcoma: A Japanese Musculoskeletal Oncology Group (JMOG) study. Cancer 2016;122:1408-16.

60. Ray-Coquard I, Italiano A, Bompas E, et al. Sorafenib for patients with advanced angiosarcoma: a phase II Trial from the French Sarcoma Group (GSF/GETO). Oncologist 2012;17:260-6.

61. Park MS, Patel SR, Ludwig JA, et al. Activity of temozolomide and bevacizumab in the treatment of locally advanced, recurrent, and metastatic hemangiopericytoma and malignant solitary fibrous tumor. Cancer 2011;117:4939-47.

62. Loizos N, Xu Y, Huber J, et al. Targeting the plateletderived growth factor receptor alpha with a neutralizing human monoclonal antibody inhibits the growth of tumor xenografts: implications as a potential therapeutic target. Mol Cancer Ther 2005;4:369-79.

63. Martin RC 2nd, Osborne DL, Edwards MJ, et al. Giant cell tumor of tendon sheath, tenosynovial giant cell tumor, and pigmented villonodular synovitis: defining the presentation, surgical therapy and recurrence. Oncol Rep 2000;7:413-9.

64. Ravi V, Wang WL, Lewis VO. Treatment of tenosynovial giant cell tumor and pigmented villonodular synovitis. Curr Opin Oncol 2011;23:361-6.

65. West RB, Rubin BP, Miller MA, et al. A landscape effect in tenosynovial giant-cell tumor from activation of CSF1 expression by a translocation in a minority of tumor cells. Proc Natl Acad Sci U S A 2006;103:690-5.

66. Cupp JS, Miller MA, Montgomery KD, et al. Translocation and expression of CSF1 in pigmented villonodular synovitis, tenosynovial giant cell tumor, rheumatoid arthritis and other reactive synovitides. Am J Surg Pathol 2007;31:970-6.
67. Patwardhan PP, Surriga O, Beckman MJ, et al. Sustained inhibition of receptor tyrosine kinases and macrophage depletion by PLX3397 and rapamycin as a potential new approach for the treatment of MPNSTs. Clin Cancer Res 2014;20:3146-58.

68. Cassier PA, Gelderblom H, Stacchiotti S, et al. Efficacy of imatinib mesylate for the treatment of locally advanced and/or metastatic tenosynovial giant cell tumor/pigmented villonodular synovitis. Cancer 2012;118:1649-55.

69. Brahmi M, Vinceneux A, Cassier PA. Current Systemic Treatment Options for Tenosynovial Giant Cell Tumor/ Pigmented Villonodular Synovitis: Targeting the CSF1/ CSF1R Axis. Curr Treat Options Oncol 2016;17:10.

70. Tan DS, Bedard PL, Kuruvilla J, et al. Promising SINEs for embargoing nuclear-cytoplasmic export as an anticancer strategy. Cancer Discov 2014;4:527-37.

71. Fung HY, Chook YM. Atomic basis of CRM1-cargo recognition, release and inhibition. Semin Cancer Biol 2014;27:52-61.

72. Huang WY, Yue L, Qiu WS, et al. Prognostic value of CRM1 in pancreas cancer. Clin Invest Med 2009;32:E315.

73. Noske A, Weichert W, Niesporek S, et al. Expression of the nuclear export protein chromosomal region maintenance/exportin 1/Xpo1 is a prognostic factor in human ovarian cancer. Cancer 2008;112:1733-43.

74. Shen A, Wang Y, Zhao Y, et al. Expression of CRM1 in human gliomas and its significance in p27 expression and clinical prognosis. Neurosurgery 2009;65:153-9; discussion 9-60.

75. Nakayama R, Zhang YX, Czaplinski JT, et al. Preclinical activity of selinexor, an inhibitor of XPO1, in sarcoma. Oncotarget 2016;7:16581-92.

76. Conyers R, Young S, Thomas DM. Liposarcoma: molecular genetics and therapeutics. Sarcoma 2011;2011:483154.

77. Dalal KM, Kattan MW, Antonescu CR, et al. Subtype specific prognostic nomogram for patients with primary liposarcoma of the retroperitoneum, extremity, or trunk. Ann Surg 2006;244:381-91.

78. Crago AM, Singer S. Clinical and molecular approaches to well differentiated and dedifferentiated liposarcoma. Curr Opin Oncol 2011;23:373-8.

79. Sreekantaiah C, Karakousis CP, Leong SP, et al. Cytogenetic findings in liposarcoma correlate with histopathologic subtypes. Cancer 1992;69:2484-95.

80. Tap WD, Eilber FC, Ginther C, et al. Evaluation of welldifferentiated/de-differentiated liposarcomas by highresolution oligonucleotide array-based comparative 
genomic hybridization. Genes Chromosomes Cancer 2011;50:95-112.

81. Singer S, Socci ND, Ambrosini G, et al. Gene expression profiling of liposarcoma identifies distinct biological types/subtypes and potential therapeutic targets in welldifferentiated and dedifferentiated liposarcoma. Cancer Res 2007;67:6626-36.

82. Barretina J, Taylor BS, Banerji S, et al. Subtype-specific genomic alterations define new targets for soft-tissue sarcoma therapy. Nat Genet 2010;42:715-21.

83. Fry DW, Harvey PJ, Keller PR, et al. Specific inhibition of cyclin-dependent kinase 4/6 by PD 0332991 and associated antitumor activity in human tumor xenografts. Mol Cancer Ther 2004;3:1427-38.

84. Schwartz GK, LoRusso PM, Dickson MA, et al. Phase I study of PD 0332991, a cyclin-dependent kinase inhibitor, administered in 3-week cycles (Schedule 2/1). Br J Cancer 2011;104:1862-8.

85. Burgess A, Chia KM, Haupt S, et al. Clinical Overview of MDM2/X-Targeted Therapies. Front Oncol 2016;6:7.

86. Ito Y, Maeda D, Yoshida M, et al. Cardiac intimal sarcoma with PDGFRbeta mutation and co-amplification of PDGFRalpha and MDM2: an autopsy case analyzed by whole-exome sequencing. Virchows Arch 2017;471:423-8.

87. Hang JF, Chen PC. Parosteal osteosarcoma. Arch Pathol Lab Med 2014;138:694-9.

88. Kikuchi K, Wettach GR, Ryan CW, et al. MDM2 Amplification and PI3KCA Mutation in a Case of Sclerosing Rhabdomyosarcoma. Sarcoma 2013;2013:520858.

89. Cassier PA, Lefranc A, Amela EY, et al. A phase II trial of panobinostat in patients with advanced pretreated soft tissue sarcoma. A study from the French Sarcoma Group. Br J Cancer 2013;109:909-14.

90. Kim KH, Roberts CW. Targeting EZH2 in cancer. Nat Med 2016;22:128-34.

91. Wilson BG, Wang X, Shen X, et al. Epigenetic antagonism between polycomb and SWI/SNF complexes during oncogenic transformation. Cancer Cell 2010;18:316-28.

92. Folpe AL. Selected topics in the pathology of epithelioid soft tissue tumors. Mod Pathol 2014;27 Suppl 1:S64-79.

93. Kohashi K, Oda Y, Yamamoto H, et al. Reduced expression of SMARCB1/INI1 protein in synovial sarcoma. Mod Pathol 2010;23:981-90.

94. Noujaim J, Thway K, Bajwa Z, et al. Epithelioid Sarcoma: Opportunities for Biology-Driven Targeted Therapy. Front Oncol 2015;5:186.

95. Schoffski P, Agulnik M, Stacchiotti S, et al. Phase 2 multicenter study of the EZH2 inhibitor tazemetostat in adults with synovial sarcoma (NCT02601950). J Clin Oncol 2017;35:11057-.

96. Vaishnavi A, Le AT, Doebele RC. TRKing down an old oncogene in a new era of targeted therapy. Cancer Discov 2015;5:25-34.

97. Chiang S, Cotzia P, Hyman DM, et al. NTRK Fusions Define a Novel Uterine Sarcoma Subtype With Features of Fibrosarcoma. Am J Surg Pathol 2018;42:791-8.

98. Blumenthal GM, Mansfield E, Pazdur R. Next-Generation Sequencing in Oncology in the Era of Precision Medicine. JAMA Oncol 2016;2:13-4.

99. Sheikine Y, Kuo FC, Lindeman NI. Clinical and Technical Aspects of Genomic Diagnostics for Precision Oncology. J Clin Oncol 2017;35:929-33.

100. Chakravarty D, Gao J, Phillips SM, et al. OncoKB: A Precision Oncology Knowledge Base. JCO Precis Oncol 2017;2017.

101. Gounder MM, Ali SM, Robinson V, et al. Impact of next-generation sequencing (NGS) on diagnostic and therapeutic options in soft-tissue and bone sarcoma. J Clin Oncol 2017;35:11001.

102.Lucchesi C, Khalifa E, Laizet Y, et al. Targetable Alterations in Adult Patients With Soft-Tissue Sarcomas: Insights for Personalized Therapy. JAMA Oncol 2018. [Epub ahead of print].

103. Comprehensive and Integrated Genomic Characterization of Adult Soft Tissue Sarcomas. Cell 2017;171:950-65.e28.

104.Chapman PB, Hauschild A, Robert C, et al. Improved survival with vemurafenib in melanoma with BRAF V600E mutation. N Engl J Med 2011;364:2507-16.

105.Prasad V. Perspective: The precision-oncology illusion. Nature 2016;537:S63.

106. Andrews A. ASCO and NCI Launch Largest Precision Medicine Trials Using Real-World Evidence. Am Health Drug Benefits 2015;8:37.

107. Harris L, Chen A, O'Dwyer P, et al. Abstract B080: Update on the NCI-Molecular Analysis for Therapy Choice (NCI-MATCH/EAY131) precision medicine trial. Mol Cancer Ther 2018;17:B080.

108. Coyne GO, Takebe N, Chen AP. Defining precision: The precision medicine initiative trials NCI-MPACT and NCI-MATCH. Curr Probl Cancer 2017;41:182-93.

109. Ribas A, Wolchok JD. Cancer immunotherapy using checkpoint blockade. Science 2018;359:1350-5.

110.McCarthy EF. The toxins of William B. Coley and the treatment of bone and soft-tissue sarcomas. Iowa Orthop J 2006;26:154-8. 
111. Bhatia K, Shiels MS, Berg A, et al. Sarcomas other than Kaposi sarcoma occurring in immunodeficiency: interpretations from a systematic literature review. Curr Opin Oncol 2012;24:537-46.

112. Rubinstein PG, Aboulafia DM, Zloza A. Malignancies in HIV/AIDS: from epidemiology to therapeutic challenges. Aids 2014;28:453-65.

113. Kim JR, Moon YJ, Kwon KS, et al. Tumor infiltrating PD1-positive lymphocytes and the expression of PD-L1 predict poor prognosis of soft tissue sarcomas. PLoS One 2013;8:e82870.

114. Sorbye SW, Kilvaer T, Valkov A, et al. Prognostic impact of lymphocytes in soft tissue sarcomas. PLoS One 2011;6:e14611.

115.Ben-Ami E, Barysauskas CM, Solomon S, et al. Immunotherapy with single agent nivolumab for advanced leiomyosarcoma of the uterus: Results of a phase 2 study. Cancer 2017;123:3285-90.

116. George S, Miao D, Demetri GD, et al. Loss of PTEN Is Associated with Resistance to Anti-PD-1 Checkpoint Blockade Therapy in Metastatic Uterine Leiomyosarcoma. Immunity 2017;46:197-204.

117.Larkin J, Chiarion-Sileni V, Gonzalez R, et al. Combined Nivolumab and Ipilimumab or Monotherapy in Untreated Melanoma. N Engl J Med 2015;373:23-34.

118. Motzer RJ, Tannir NM, McDermott DF, et al. Nivolumab plus Ipilimumab versus Sunitinib in Advanced Renal-Cell Carcinoma. N Engl J Med 2018;378:1277-90.

119. Ge Y, Domschke C, Stoiber N, et al. Metronomic cyclophosphamide treatment in metastasized breast cancer patients: immunological effects and clinical outcome. Cancer Immunol Immunother 2012;61:353-62.

120. Toulmonde M, Penel N, Adam J, et al. Use of PD-1 Targeting, Macrophage Infiltration, and IDO Pathway Activation in Sarcomas: A Phase 2 Clinical Trial. JAMA Oncol 2018;4:93-7.

121. Chen DS, Mellman I. Elements of cancer immunity and the cancer-immune set point. Nature 2017;541:321-30.

122.Pollack SM, He Q, Yearley JH, et al. T-cell infiltration and clonality correlate with programmed cell death protein 1 and programmed death-ligand 1 expression in patients with soft tissue sarcomas. Cancer 2017;123:3291-304.

123. Khan KA, Kerbel RS. Improving immunotherapy outcomes with anti-angiogenic treatments and vice versa. Nat Rev Clin Oncol 2018;15:310-24.

124. Hodi FS, Lawrence D, Lezcano C, et al. Bevacizumab plus ipilimumab in patients with metastatic melanoma. Cancer Immunol Res 2014;2:632-42.
125. Wallin JJ, Bendell JC, Funke R, et al. Atezolizumab in combination with bevacizumab enhances antigen-specific T-cell migration in metastatic renal cell carcinoma. Nat Commun 2016;7:12624.

126. Atkins MB, Plimack ER, Puzanov I, et al. Axitinib in combination with pembrolizumab in patients with advanced renal cell cancer: a non-randomised, open-label, dose-finding, and dose-expansion phase $1 \mathrm{~b}$ trial. Lancet Oncol 2018;19:405-15.

127. Wilky BA, Trucco MM, Kolonias D, et al. A phase II trial of axitinib plus pembrolizumab for patients with advanced alveolar soft part sarcoma (ASPS) and other soft tissue sarcomas (STS). J Clin Oncol 2018;36:11547.

128. Salah S, Lewin JH, Davidson S, et al. Immunoprofiling in alveolar soft part sarcoma. J Clin Oncol 2017;35:11059.

129.Le DT, Durham JN, Smith KN, et al. Mismatch repair deficiency predicts response of solid tumors to PD-1 blockade. Science 2017;357:409-13.

130.D'Angelo SP, Shoushtari AN, Agaram NP, et al. Prevalence of tumor-infiltrating lymphocytes and PD-L1 expression in the soft tissue sarcoma microenvironment. Hum Pathol 2015;46:357-65.

131. Bellmunt J, de Wit R, Vaughn DJ, et al. Pembrolizumab as Second-Line Therapy for Advanced Urothelial Carcinoma. N Engl J Med 2017;376:1015-26.

132. Herbst RS, Soria JC, Kowanetz M, et al. Predictive correlates of response to the anti-PD-L1 antibody MPDL3280A in cancer patients. Nature 2014;515:563-7.

133. Rosenberg SA, Restifo NP. Adoptive cell transfer as personalized immunotherapy for human cancer. Science 2015;348:62-8.

134. Gjerstorff MF, Andersen MH, Ditzel HJ. Oncogenic cancer/testis antigens: prime candidates for immunotherapy. Oncotarget 2015;6:15772-87.

135.Pollack SM, Jungbluth AA, Hoch BL, et al. NY-ESO-1 is a ubiquitous immunotherapeutic target antigen for patients with myxoid/round cell liposarcoma. Cancer 2012;118:4564-70.

136. Endo M, de Graaff MA, Ingram DR, et al. NY-ESO-1 (CTAG1B) expression in mesenchymal tumors. Mod Pathol 2015;28:587-95.

137.Iura K, Maekawa A, Kohashi K, et al. Cancer-testis antigen expression in synovial sarcoma: NY-ESO-1, PRAME, MAGEA4, and MAGEA1. Hum Pathol 2017;61:130-9.

138. Constantino J, Gomes C, Falcao A, et al. Dendritic cellbased immunotherapy: a basic review and recent advances. Immunol Res 2017;65:798-810.

139. Wang H, Zhang L, Kung SK. Emerging applications of 
lentiviral vectors in dendritic cell-based immunotherapy. Immunotherapy 2010;2:685-95.

140. Pollack SM. The potential of the CMB305 vaccine regimen to target NY-ESO-1 and improve outcomes for synovial sarcoma and myxoid/round cell liposarcoma patients. Expert Rev Vaccines 2018;17:107-14.

141. Dudley ME, Wunderlich JR, Robbins PF, et al. Cancer

Cite this article as: Yen CC, Chen TW. Next frontiers in systemic therapy for soft tissue sarcoma. Chin Clin Oncol 2018;7(4):43. doi: 10.21037/cco.2018.08.04 regression and autoimmunity in patients after clonal repopulation with antitumor lymphocytes. Science 2002;298:850-4.

142. González-Galarza FF, Takeshita LY, Santos EJ, et al. Allele frequency net 2015 update: new features for HLA epitopes, KIR and disease and HLA adverse drug reaction associations. Nucleic Acids Res 2015;43:D784-8. 